\title{
МОДЕЛИРОВАНИЕ ПРОЦЕССА РАЗРЫВА ЭЛЕКТРИЧЕСКОЙ ДУГИ В ЭЛЕКТРОДУГОВОЙ СТАЛЕПЛАВИЛЬНОЙ ПЕЧИ
}

\author{
Михайлов Василий Александрович \\ Рыбинский государственный авиачионный \\ технический университет имени П.А. Соловьева, \\ 2. Рыбинск \\ Zepewb5@gmail.com
}

Аннотация. Сталеплавильное производство - быстроразвивающаяся отрасль промышленности. Современные условия требуют от производителей максимальных объемов выработки при минимальных инвестиционных составляющих. При этом возникает необходимость научных исследований процесса появления электрической дуги и методов ее ликвидации.

Ключевые слова: Электрическая дуга, сталеплавильная печь, характеристики, параметры, математические модели, моделирование, ток, напряжение.

$\Delta Q_{\beta}$ - непрогнозируемые параметры; ного производства предъявляют высокие требования к усовершенствованию процессов управления разрывом электрической дуги в электродуговых сталеплавильных печах (ДСП) и создания математических моделей сталеплавильного производства. Математическая модель ДСП не только воссоздает условия процесса плавки, но и описывает входные и выходные данные, которые оказывают влияние на оперативное обслуживание и промышленную эксплуатацию реального объекта. Сделанные выводы помогают произвести годовое, среднесрочное и стратегическое планирование дальнейшего развития производства.

На рисунке 1 представлена структурированная схема ДСП как самостоятельного объекта в системе управления.

Условные обозначения на рисунке 1:

$U_{2}$ - напряжение на вторичной обмотке трансформатора;

$U_{\phi}$ - фазное напряжения;

$L_{\text {дуга }}$ - длина дуги;

$I_{\text {дуа }}$ - электрической ток;

$\Delta L_{\text {дуга }}$ - хаотичные факторы;
$B$-факторы, зависящие от схемы питающих линий внешнего электроснабжения.

Следует сказать, что параметры ДСП прямопропорционально зависят от характеристик электрической дуги (ЭД), в частности от ее мощности, которая воздействует на условия работы установки. Расплавление происходит в несколько этапов. Каждый этап имеет свой режим работы электрической сети и поглощаемой мощности. К внешним факторам относятся напряжение на вторичной обмотке трансформатора и межэлектродное расстояние - длина ЭД [6, с. 1812-1817, 8, с. 704-711, 9, c. 653-663].

Вышеприведенная математическая модель поверхностно описывает внутренние процессы, происходящие в ДСП. Построение более точной модели должно сопровождаться анализом физических параметров, связанных с электрическими переходами.

Метод, основанный на схеме замещения трехфазной ЭД в электрической цепи без учета нулевого провода, позволяет изучить параметры ДСП. Для сокращения 


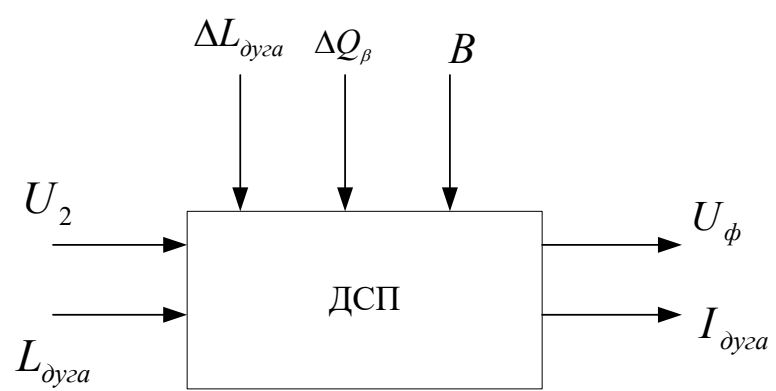

Рис. 1. Структурированная схема ДСП

объема вычислений вводится допущение: активные сопротивления и индуктивности всех элементов электрической цепи принимаются постоянными и независимыми величинами.

Формульное значение структурированной схемы, представленной на рисунке 1 примет следующий вид [3, с. 124-129]:

$U_{1}^{\prime} \cdot \sin \omega t-U_{1}-U_{0}=r_{1} \cdot i_{1}(t)+L_{1} \cdot \frac{d i(t)}{d t}$

$U_{2}^{\prime} \cdot \sin \left(\omega t-120^{\circ}\right)+U_{2}-U_{0}=$

$=r_{2} \cdot i_{2}(t)+L_{2} \cdot \frac{d i(t)}{d t}$,

$U_{3}^{\prime} \cdot \sin \omega t-U_{3}-U_{0}=r_{3} \cdot i_{3}(t)+L_{3} \cdot \frac{d i(t)}{d t}$

$i_{1}(t)+i_{2}(t)+i_{3}(t)=0$,

где $U_{1}^{\prime}, U_{2}^{\prime}, U_{3}^{\prime}-$ пофазная разность потенциалов;

$U_{1}, U_{2}, U_{3}-$ разность потенциалов ЭД;

$U_{0}$ - мгновенная разность потенциалов в точке нулевого схождения ЭД;

$r_{1}, r_{2}, r_{3}$ - цифровое выражение активного сопротивления;

$L_{1}, L_{2}, L_{3}$ - цифровое выражение реактивного сопроивления;

$i_{1}, i_{2}, i_{3}$ - электрическая сила тока дуги.

Научные опыты доказали верность следующего математического выражения:

$U_{\text {дуга }}=\alpha+\beta \cdot l$

где $\alpha$ - суммарное значение падения напряжения;

$\beta$ - градиент потенциала электрической дуги, характеризующий состав, температуру и давление газа.

Самый сложный процесс - составление математической модели изменения состояния ЭД. Ее заменяют активной переменной составляющей полного сопротивления. При таком расчете невозможно на 100\% исследовать нелинейные характеристики ЭД.

Часть исследователей поддерживают идею по развитию теории электрической проводимости дуги. Например, нелинейное дифференциальное уравнение Кассии $[5$, с. $1-14 ; 7$, с. 35-39]:

$$
\frac{d g_{\text {дуга }}(t)}{d t}=\frac{1}{\Theta} \cdot\left(\frac{i_{\text {дуга }}^{2}}{U_{\text {дуга }}^{2} g_{\text {дуга }}}-g_{\text {дуга }}\right),
$$

где $\Theta$ - постоянный коэффициент;

$i_{\text {дуа }}$ - цифровое выражение силы электрического тока;

$U_{\text {дуга }}$ - амплитудная разность потенциалов;

$g_{\text {дуга }}-$ электрическая проводимость.

Макет, полученный из вышеуказанного математического выражения, предусматривает нелинейные свойства ЭД в различные фазы горения.

При наличии общей схемы замещения расчетным путем находятся числовые параметры используемых величин [2, с. 23-25; 4, с. 245-256; 10, с. 507-511]. Приведем пример расчета сопротивлений.

Суммарное активное сопротивление является суммой активных сопротивлений участков электрической цепи:

$$
R=R_{\text {иина }}+R_{\text {кабель }}+R_{\text {труба }}+R_{\text {контакт }}+R_{\text {электрод }}
$$

где $R_{\text {шина }}$ - активное сопротивление шины;

$R_{\text {кабель }}$ - активное сопротивление провода;

$R_{\text {mруба }}$ - активное сопротивление изолирующей конструкции;

$R_{\text {контакт }}$ - активное сопротивление электроконтакта;

$R_{\text {электрод }}$ - активное сопротивление сетки.

Формульное выражение

$R=\rho_{0} \cdot\left[1+\alpha_{r} \cdot\left(T-T_{0}\right)\right] \cdot \frac{l}{S}$, 


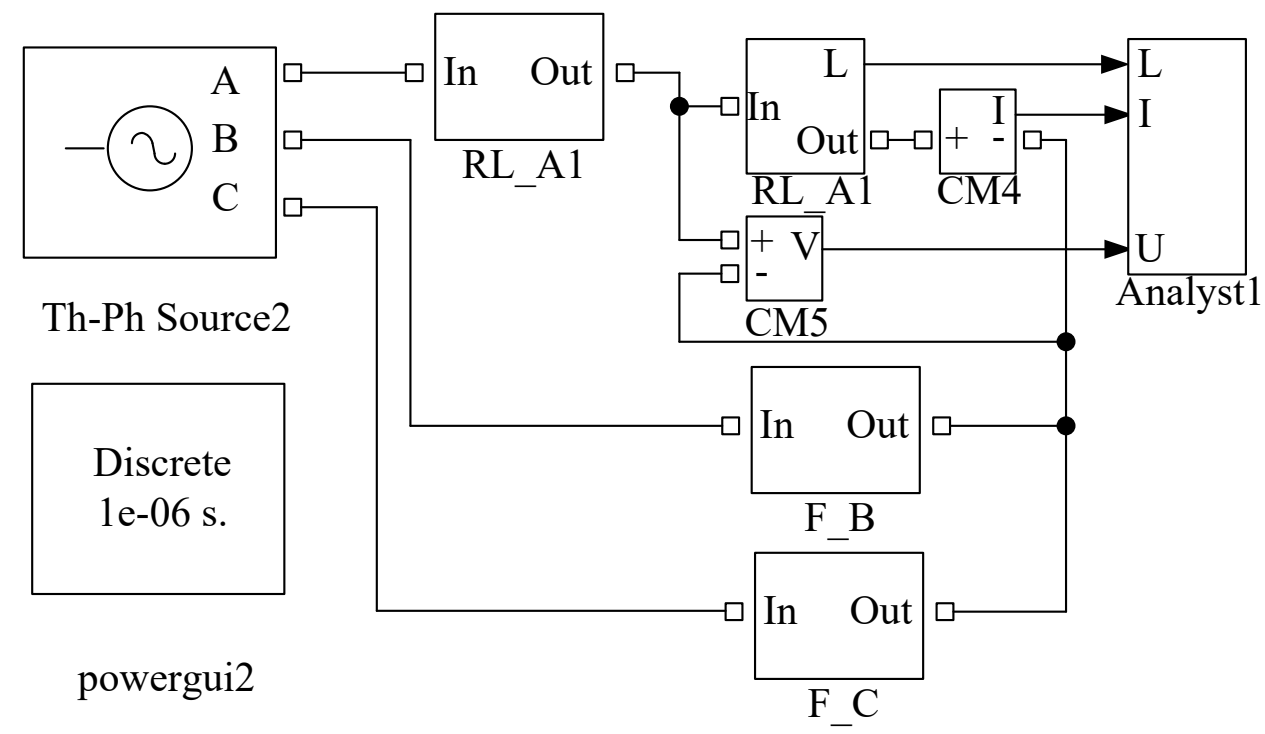

Рис. 2. Обобщенный макет ДСП

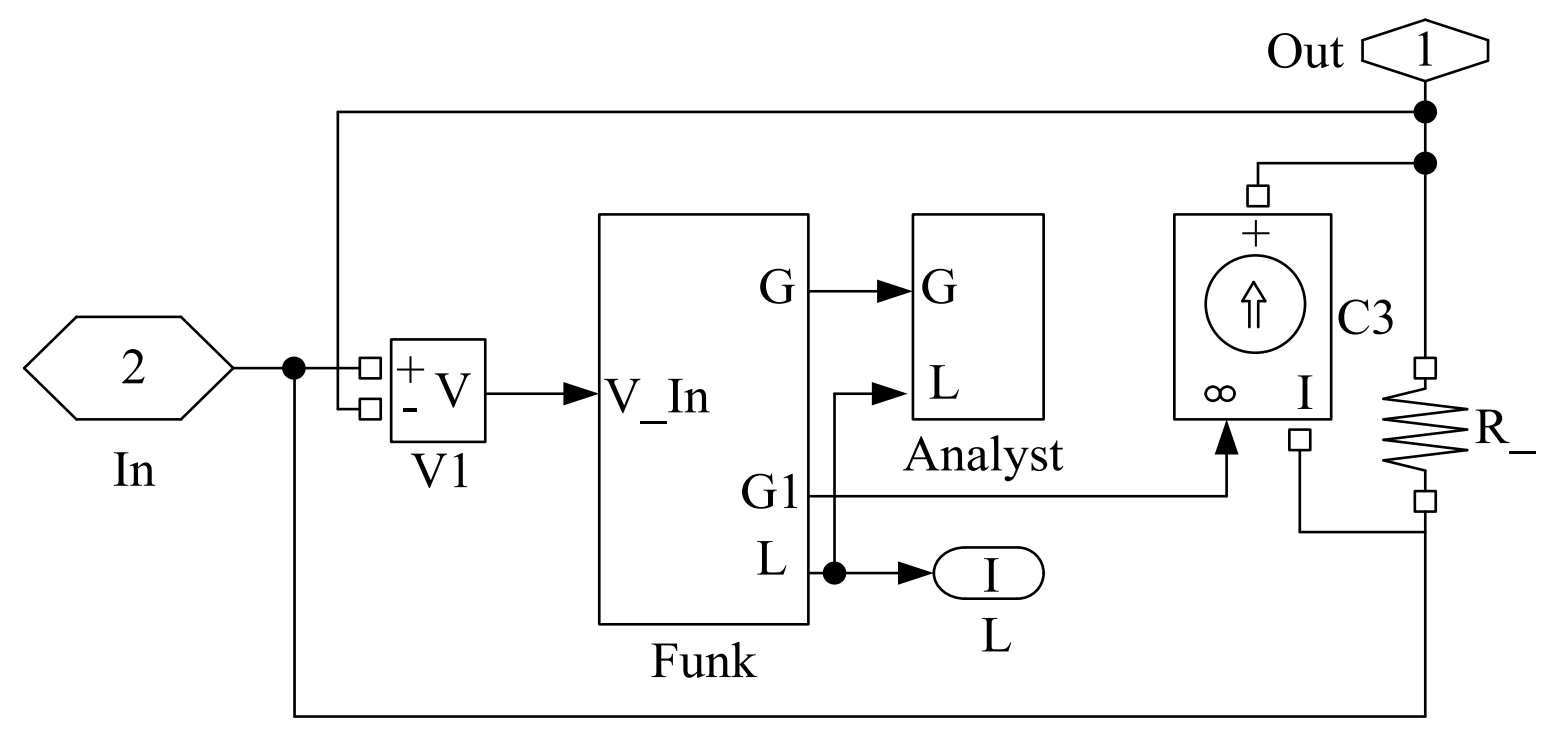

Рис. 3. Выделенная модель «Arc_x»

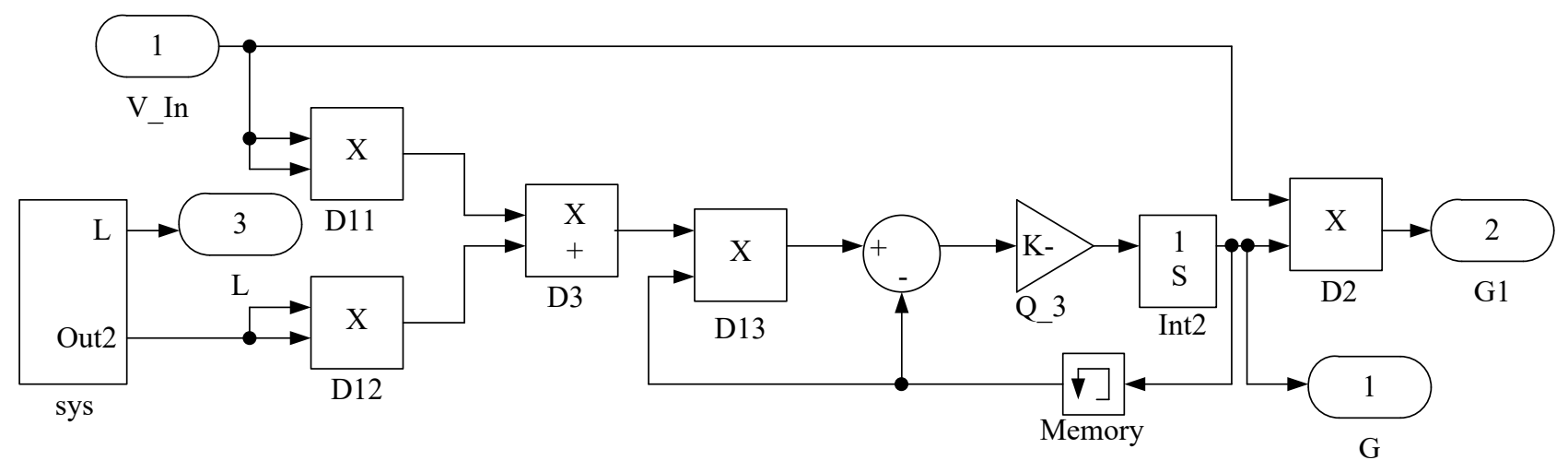

Рис. 4. Выделенная модель «Funk» 


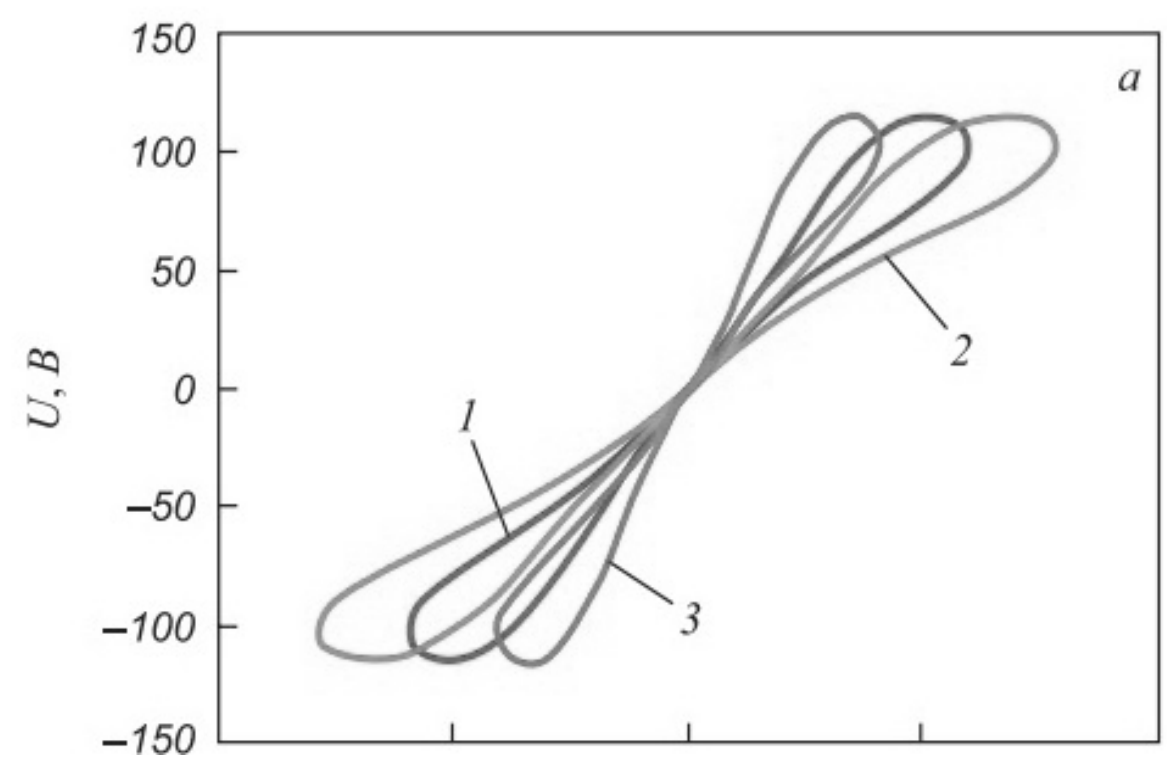

Рис. 5а. ВАХ при напряжении на вторичной обмотке трансформатора (1-115 B, 2-127 B, 3-104 B)

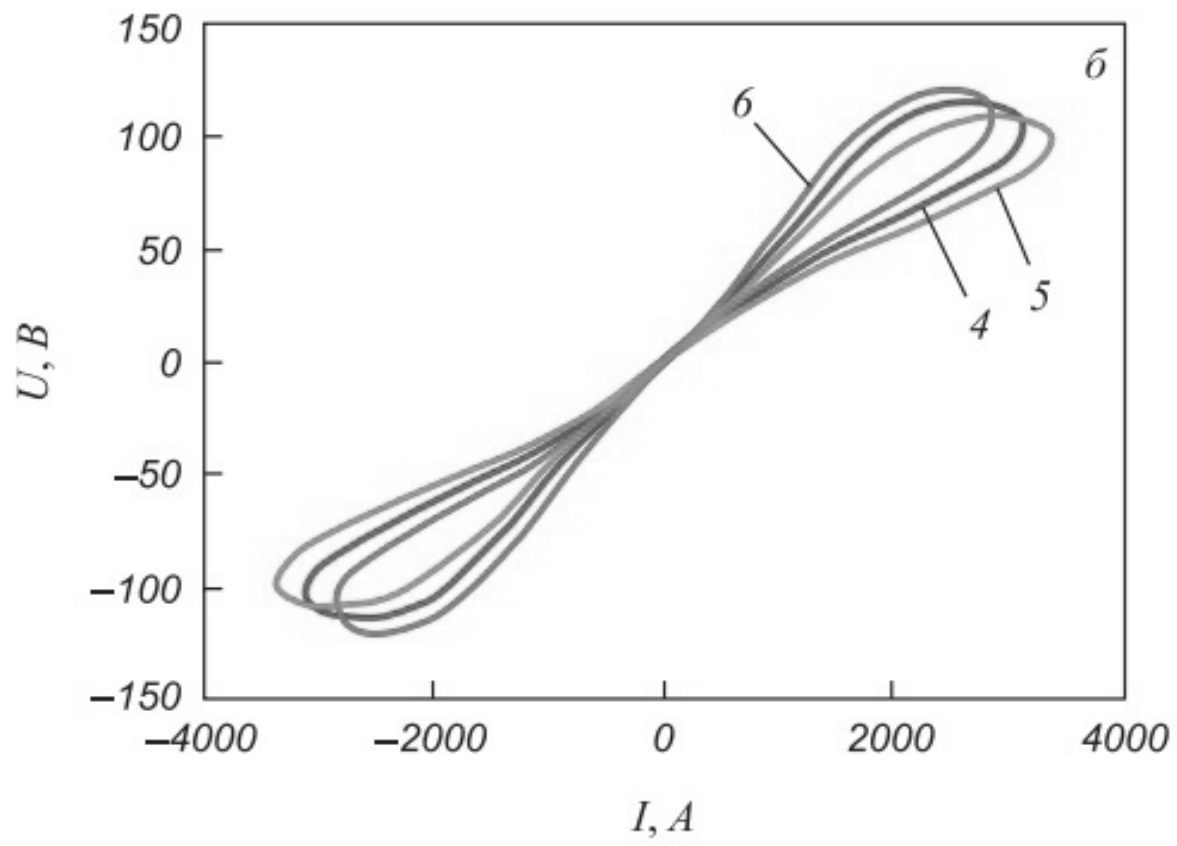

Рис. 56. ВАХ для различных длин ЭД (4-0,02 м, 5-0,018 м, 6-0,022 м)

где $\rho_{0}$-удельное сопротивление материала проводника при абсолютном нуле;

$\alpha_{r}$ - температурная постоянная;

$T$ - текущее значении температуры;

$T_{0}$ - абсолютный нуль;

$l$ - длина образца;

$S$ - площадь поперечного сечения образца.
Каждый участок электрической цепи представляет собой простейший элемент, сопротивление которого находится по вышеприведенному уравнению.

Общее индуктивное сопротивление[1, с. 27-28]:

$$
L=L_{\text {пакет }}+L_{\text {участок }}+L_{\text {кабель }}+L_{\text {труба }}+L_{\text {электрод }} \text {, }
$$




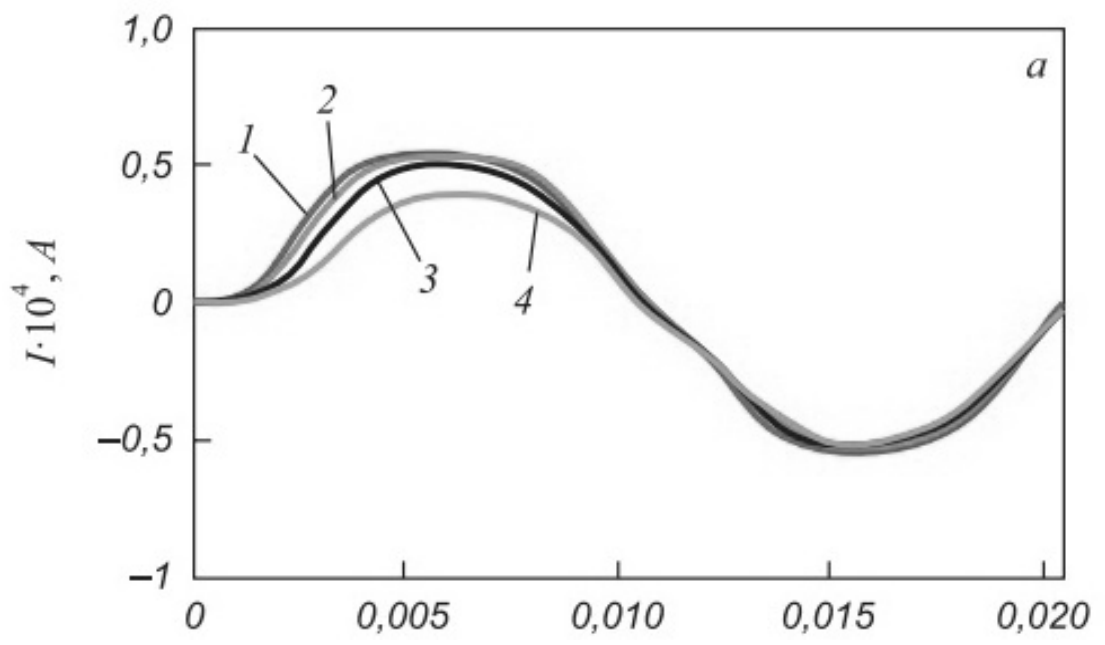

Рис. 6а. Осциллограммы тока

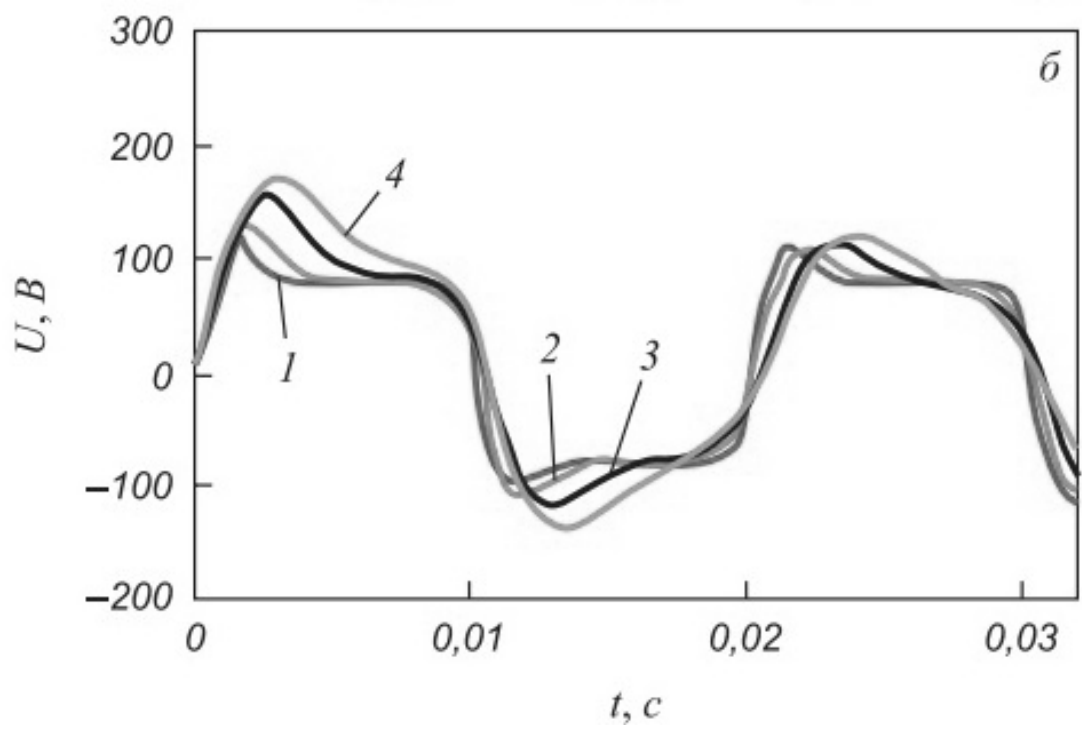

Рис. 66. Осциллограммы напряжения

где $L_{\text {пакет }}-$ индуктивность шинного пакета;

$L_{\text {участок }}$ - индуктивность участка расшихтовки;

$L_{\text {кабель }}$ - индуктивность кабеля;

$L_{\text {труба }}$ - индуктивность трубы;

$L_{\text {электрод }}$ - индуктивность электрода.

После проведения математических операций начинается процесс моделирования. Процесс моделирования произведен в программном комплексе Matlab Simulink. Структура математической модели представлена на рисунках 2-4.

Блок-схема (рис. 2) состоит из следующих блоков:
- агрегат переменной разности потенциалов «ThPhSource2», выдающий напряжение, равное напряжению вторичной обмотке трансформатора;

- блок активно-реактивных сопротивлений;

- три блока электрической дуги каждой фазы «D_ $A 1 », « F \_B », « F \_C »$.

Блок-схема (рис. 3) представляет собой блоки-модуляторы:

- «V1»- блок измерения мгновенного значения напряжения;

- «СЗ»-источник-преобразователь тока; 


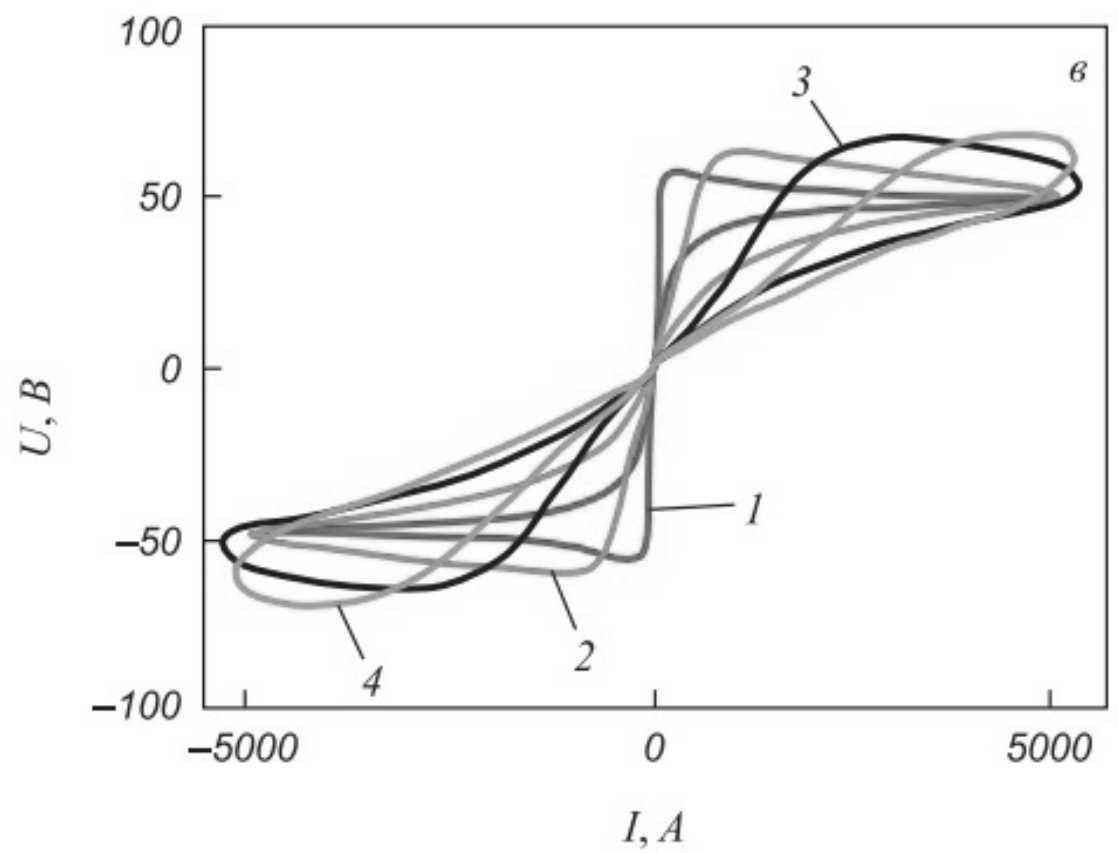

Рис. 6в. ВАХ ЭД

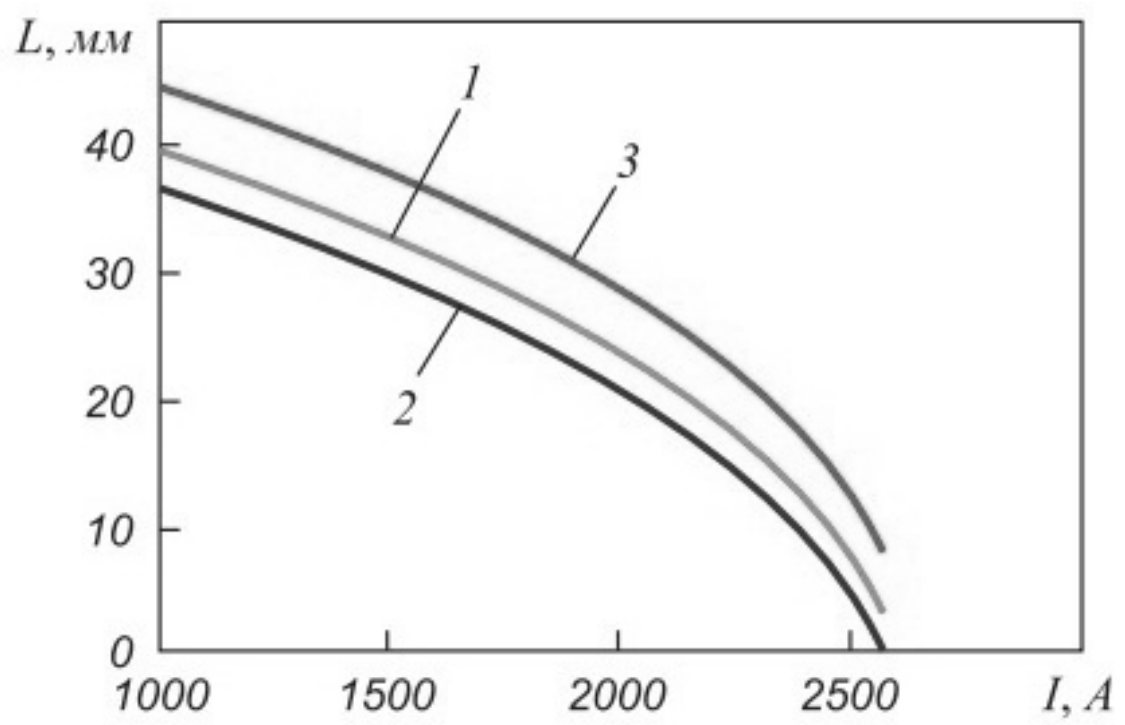

Рис. 7. Зависимость электротока и длины ЭД при изменяющейся разности потенциалов (1-110 B, 2-104 B, 3-120 B)

४ «R»-дополнительное сопротивление, применяемое для гальванической развязки;

- «Func»-модель электрической дуги, которая выстаивает вольт-амперную характеристику (BAX) объекта наблюдения;

- «Analyst»-анализирующий блок, выдает графические зависимости для последующего анализа.
Блок-схема (рис. 4) представляет внутренние компоненты блока «Func», использующие уравнение Кассии, а также блоки суммы, интегральные и дифференцирующие блоки.

Для проведения углубленного анализа необходимо учитывать не только статические, но и динамические параметры ДСП. 
Самый важный динамический параметр - BAX. Форма ВАХ указывает на характеристики ДСП.

Далее формируются BAX для конкретных значений разности потенциалов на вторичной обмотке трансформатора (рис. 5а) и длины ЭД (рис. 56).

На ДСП оказывают влияние внешние нерегулируемые факторы. На рисунке 6(а, 6, в) представлены осциллограммы напряжения и тока, полученные на основании динамических BAX с учетом изменяющейся «постоянной времени проводимости электрической дуги» (1-0,00015 с, 2-0,0006 с, 3-0,002 c, 4-0,005 c). Развитие ионизации нестабильно на первом этапе расплавления, «постоянная времени» приравнивается 0,00015 с. Стадия окидировки - 0,002 с. Стадия рафинирования - 0,005 с. Стадия окончания плавки -0,0006 с.
Не менее важны статические параметры ДСП, показывающие влияние электротока на длину ЭД. Этот процесс является нелинейным (рис. 7).

Проанализировав рисунки 5а, 56 стоит отметить невозможность использование регулировки напряжения на вторичной обмотке трансформатора ввиду технологических особенностей оборудования ДСП.

Анализ рисунков 6а,6б,бв доказывает необходимость ограничения потребляемой мощности ДСП.

Таким образом, материал, представленный в данной работе, доказал, что процессы, происходящие в ДСП достаточно непростые и малоизученные, а ДСП - функционально сложные и технологически разнообразные промышленные объекты.

\section{ЛИТЕРАТУРА}

1. Данцис Я.Б., Кацевич Л. С., Жилов Г. М. Короткие сети и электрические параметры дуговых электропечей. М.: Металлургия, 1987. 320 с.

2. Егоров А. В. Электроплавильные печи черной металлургии. М.: Металлургия, 1985. 280 с.

3. Свенчанский А.Д., Жердев И. Т., Кручинин А. М. Электрические промышленные печи: Дуговые печи и установки специального нагрева. М.: Энергоиздат, 1981. 296 c.

4. Чередниченко В.С., Аньшаков А.С., Кузьмин М. Г. Плазменные электротехнологические установки. Новосибирск: Изд-во НГтУ, 2011.602 с.

5. Cassie A.M. A new theory of arc rupture and circuit Severity // CIGRE. 1939. No. 102. P. 1-14.

6. Collantes-Bellido R., Gomez T. Identification and modeling of a three phase arc furnace for voltage disturbance simulation // IEEE Transactions on Power Delivery. 1997. Vol. 12. No. 4. P. 1812-1817.

7. Gelada J. Electrical analysis of the steel melting arc furnace // Iron and Steel Engineer. 1993. Vol. 70. No. 5. P. 35-39.

8. Heydt G.T., O'Neill-Carrillo E., Zhao R.Y. The modeling of nonlinear loads as chaotic systems in electric power engineering // Proc. of the IEEE/PEC International Conference on Harmonics and Quality of Power. Las Vegas. 1996. P. 704-711.

9. Higgs R. W. Sonic signature analysis for arc furnace diagnostics and control // Proc. of Ultrasonics Symposium. Milwaukee. 1974. P. 653-663

10. Ozgun 0., Abur A. Development of an arc furnace model for power quality studies // Power Engineering Society Summer Meeting. 1999. No. 1. P. $507-511$. 